\title{
Vascular Endothelial Growth Factor Inhibitor-Induced Hypertension: Basics for Primary Care Providers
}

\author{
Carmen P. Escalante and Ali Zalpour \\ Department of General Internal Medicine, University of Texas MD Anderson Cancer Center, Unit 1465, Houston, \\ TX 77230-1402, USA \\ Correspondence should be addressed to Carmen P. Escalante, cescalan@mdanderson.org
}

Received 22 November 2010; Revised 2 March 2011; Accepted 5 March 2011

Academic Editor: Hans R. Brunner

Copyright $\odot 2011$ C. P. Escalante and A. Zalpour. This is an open access article distributed under the Creative Commons Attribution License, which permits unrestricted use, distribution, and reproduction in any medium, provided the original work is properly cited.

\begin{abstract}
Frequently, primary care providers continue to manage the overall medical care of cancer patients. With newer and often more potent antitumor agents, patients may present to their local physicians with drug-induced toxicities such as hypertension induced by vascular endothelial growth factor (VEGF) inhibitors. It is imperative that these healthcare providers are aware of basic aspects of this drug class, as its use has increased significantly in the last several years. Uncontrolled or malignant hypertension due to these agents should be recognized readily and treated early to prevent more severe outcomes. This overview provides a brief background on the role of VEGF and angiogenesis in tumor metabolism as well as theories of the mechanism of VEGF inhibitors and hypertension. Helpful clinical practice aspects including the types of inhibitors used in the United States and their pharmacologic characteristics will be discussed. Also, diagnosis and treatment of hypertension induced by vascular endothelial growth factors are reviewed. A summary of key aspects of this drug class and hypertension is included.
\end{abstract}

\section{Introduction}

Cancer treatment continues to advance rapidly, and patients may experience side effects from newer treatments that are unfamiliar to their physicians. The intricacies of many diseases may necessitate several clinicians managing multiple diseases in an individual. The primary care physician often continues to oversee care of a patient's chronic illnesses such as hypertension or diabetes while the oncologist manages the patient's cancer treatment. With newer chemotherapy agents now available that are not similar to traditional chemotherapy agents such as VEGF inhibitors, non-oncology clinicians may not readily recognize common side effects induced by these agents.

Common cancers, such as colorectal and breast cancers are treated with these agents, VEGF inhibitors, and it is likely as their use becomes more widespread that primary care physicians will care for patients receiving such agents. Therefore, it is imperative that primary care physicians become more familiar with the VEGF inhibitors and their common side effects. One of the most important side effects associated with VEGF inhibitors is hypertension, and if left untreated, it could have detrimental cardiovascular complications such as renal damage or stroke. The objective of this review is to outline aspects of VEGF inhibitors used in the United States, including their proposed mechanism of action in inducing hypertension, the rates and severity of associated hypertension, and the current treatment recommendations for hypertension secondary to these agents.

\section{Scientific Background of Angiogenesis}

Angiogenesis is the complicated process of creating new blood vessels. Aberrant angiogenesis plays a role in certain diseases, including diabetes, psoriasis, rheumatoid arthritis, and various malignancies $[1,2]$. Neoangiogenesis is a significant factor in a tumor's ability to grow and metastasize [3].

During tumor growth, there are two commonly described phases: a prevascular phase and a vascular phase. During the prevasculature phase, the tumor grows by 
utilizing nutrients and oxygen obtained from the host's vasculature. In general, cell propagation and death are in balance and tumor expansion is minimal and does not exceed more than a few millimeters. Tumor neovasculature differs from normal blood vasculature by being irregular, heterogeneous, and leaky, and by showing no clear difference between arterioles and venules. Endothelial cells tend to be irregularly shaped with frequent overlap and an overall disorganized appearance [4]. During the vascular phase (or neoangiogenesis), there is brisk amplification of size and metastatic capability [5]. Several factors, such as, tumor type, cytokines, and proangiogenic genes can stimulate the tumor from a dormant to an invasive state, "angiogenic switch" [6].

\section{Angiogenesis Inhibitors}

Angiogenesis inhibitors block tumor angiogenesis and target vascular endothelial cells. Several factors favor the use of these agents over the use of conventional chemotherapeutic agents. Because endothelial tumor cells are growing and moving rapidly, angiogenesis inhibitors may be a more effective treatment option than conventional chemotherapeutic agents [7]. Endothelial cells tend to be more uniform in composition and have greater stability as a target than would a diverse array of malignant cells [8]. Angiogenesis inhibitors can readily reach the endothelium of malignant cells by systemic administration. Synergistic effects can be produced when some angiogenesis inhibitors are combined with cytotoxic drugs and radiation therapy [9].

Angiogenesis can be inhibited by targeting different mechanisms. Agents may be administered to bind angiogenic factors (e.g., VEGF), block angiogenic factor receptors (antiVEGF-receptor antibodies), interrupt intracellular signaling pathways (tyrosine kinase inhibitors), and mimic endogenous angiogenesis inhibitors (angiostatin-1,-2,endostatin, thrombospondin-1).

\section{Vascular Endothelial Growth Factor (VEGF)}

VEGF is one of the primary regulators of angiogenesis and can initiate a network of signaling processes promoting endothelial cell growth, movement, and survival following the activation of the VEGF-receptor pathway. At present, angiogenesis inhibitors are typically combined with chemotherapy. Bevacizumab was the first antiangiogenic agent approved in the United States for the treatment of colorectal cancer in combination with chemotherapy. Since then, other antiangiogenic agents have been approved to treat various malignancies (Table 1) [10-15].

\section{Hypertension and Vascular Endothelial Growth Factor (VEGF) Inhibitors}

Hypertension is a frequent adverse event of VEGF inhibitors but its incidence and severity vary by treatment agent. The overall occurrence of hypertension induced by bevacizumab, sorafenib, sunitinib, nilotinib, pazopanib, and dasatinib ranges from $9 \%$ to $67 \%$. More severe hypertension (grades
3,4 ) ranges from $3 \%$ to $18 \%$ among these agents (Table 1 ) [10-15]. Patients with underlying hypertension appear to be more at risk for severe hypertension during cancer treatment than patients without a prior diagnosis of hypertension.

Several mechanisms may induce VEGF inhibitor-related hypertension. Rarefaction, a decreased number of small arteries and arterioles, can result from treatment with VEGF kinase inhibitors and may contribute to the development of hypertension. Rarefaction of arterioles and capillaries has been noted in 6- to 8-week-old hypertensive rats with rapid microvascular rarefaction occurring after 3 days of being fed a high-salt diet [16, 17]. This finding suggests that rarefaction occurs relatively early in the development of hypertension. Microvascular rarefaction has also been shown in hypertensive adults. Steeghs et al. first demonstrated capillary rarefaction in patients treated with telatinib (BAY 57-9352), an oral inhibitor of VEGF receptors-2 and -3 (VEGFR-2, -3) [18].

A second mechanism that may contribute to hypertension is increased arterial stiffness in proximal or distal vessels. Veronese et al. [19] found that patients treated with sorafenib for 3 to 6 weeks showed a significant increase in arterial stiffness, as indicated by the central aortic augmentation index and aortic pulse wave velocity. Plasma renin, aldosterone, and catecholamine levels were not affected in those patients, demonstrating that renovascular means and volume expansion were not considered major contributors to their development of hypertension [19].

A third proposed mechanism that may contribute to hypertension is increased vascular resistance due to decreased nitric oxide and prostacyclin production. A previous study demonstrated that VEGF plays a significant role in maintaining basal vascular tone by regulating nitric oxide synthesis [20]. Wheeler-Jones et al. demonstrated that another pathway may be involved in mediating the vasoactive effects of VEGF via prostacyclin release. Preliminary results suggested that inhibiting signaling through the VEGF pathway could lead to decreased nitric oxide and prostacyclin production, thus increasing vascular resistance and blood pressure (BP) [20].

\section{Types of VEGF-Kinase Inhibitors}

The VEGF-kinase inhibitors utilized currently in clinical practice in the United States include bevacizumab, sorafenib, sunitinib, nilotinib, pazopanib, and dasatinib. Common features of these drugs/inhibitors are outlined in Table 1.

6.1. Bevacizumab. Bevacizumab was the first anti-VEGF monoclonal antibody approved for clinical practice and has been most frequently used to treat metastatic colorectal cancer. Bevacizumab is a recombinant, humanized, monoclonal antibody that binds to VEGF and prevents its association with endothelial receptors. Bevacizumab inhibits angiogenesis by VEGF binding. By inhibiting microvascular growth, bevacizumab is thought to slow the growth of all tissues, including malignant tissue. The half-life elimination of bevacizumab is 20 days, with a range of 11 to 50 days. 
TABLE 1: Current VEGF kinase inhibitors in use in the United States [10-15].

\begin{tabular}{|c|c|c|c|c|c|c|}
\hline $\begin{array}{l}\text { Name } \\
\text { (proprietary name) }\end{array}$ & $\begin{array}{l}\text { Bevacizumab } \\
\text { (Avastin) }\end{array}$ & $\begin{array}{l}\text { Sorafenib } \\
\text { (Nexavar) }\end{array}$ & $\begin{array}{l}\text { Sunitinib } \\
\text { (Sutent) }\end{array}$ & $\begin{array}{l}\text { Nilotinib } \\
\text { (Tasigna) }\end{array}$ & $\begin{array}{l}\text { Pazopanib } \\
\text { (Votrient) }\end{array}$ & $\begin{array}{l}\text { Dasatinib } \\
\text { (Sprycel) }\end{array}$ \\
\hline $\begin{array}{l}\text { Mechanism of } \\
\text { action }\end{array}$ & $\begin{array}{l}\text { Anti-VEGF } \\
\text { monoclonal } \\
\text { antibody }\end{array}$ & $\begin{array}{l}\text { Tyrosine kinase } \\
\text { inhibitor; VEGF } \\
\text { inhibitor }\end{array}$ & $\begin{array}{l}\text { Tyrosine kinase } \\
\text { inhibitor; VEGF } \\
\text { inhibitor }\end{array}$ & $\begin{array}{l}\text { Tyrosine kinase } \\
\text { inhibitor }\end{array}$ & $\begin{array}{l}\text { Tyrosine kinase } \\
\text { inhibitor; VEGF } \\
\text { inhibitor }\end{array}$ & $\begin{array}{l}\text { Tyrosine kinase } \\
\text { inhibitor }\end{array}$ \\
\hline $\begin{array}{l}\text { Hypertension } \\
\text { (\%)any grade }\end{array}$ & $23-67$ & $9-17$ & $15-30$ & $1-10$ & 40 & $1-10$ \\
\hline $\begin{array}{l}\text { Hypertension (\%) } \\
\text { grade } 3 \text { or more }\end{array}$ & $5-18$ & $\begin{array}{l}\text { Grade } 3: 3-4 \\
\text { grade } 4:<1\end{array}$ & $4-10$ & Not reported & Grade 3: 4 & Not reported \\
\hline $\begin{array}{l}\text { FDA-labeled } \\
\text { indication }\end{array}$ & $\begin{array}{l}\text { GBM } \\
\text { Metastatic breast } \\
\text { cancer } \\
\text { Metastatic } \\
\text { colorectal cancer } \\
\text { Non-small cell } \\
\text { lung cancer }\end{array}$ & $\begin{array}{l}\text { Liver carcinoma } \\
\text { Renal cell } \\
\text { carcinoma }\end{array}$ & $\begin{array}{l}\text { GI stromal tumor } \\
\text { Renal cell } \\
\text { carcinoma }\end{array}$ & CML & $\begin{array}{l}\text { Advanced renal cell } \\
\text { carcinoma }\end{array}$ & CML \\
\hline
\end{tabular}

Abbreviations: VEGF, vascular endothelial growth factor; FDA, Food and Drug Administration; GBM, glioblastoma multiforme; CML, chronic myelogenous leukemia; GI, gastrointestinal.

Bevacizumab is administered intravenously. BP monitoring is recommended every 2 to 3 weeks during bevacizumab therapy. Other cardiovascular adverse effects include arterial thromboembolism $(6 \%-9 \%)$, congestive heart failure with prior anthracycline therapy $(2 \%-4 \%)$, hypertensive encephalopathy, and myocardial infarction [10].

Bevacizumab may potentiate the cardiotoxic effects of anthracyclines. Heart failure is more common when bevacizumab is administered to patients with prior exposure to anthracyclines and/or left chest wall irradiation than to patients without these prior exposures. Proteinuria may occur with or without hypertension (any grade, 4\%-36\%) and should be monitored during treatment. Patients with a history of hypertension may have increased risk of proteinuria during treatment. Patients with a $2+$ or greater urine dipstick reading should have a 24-hour urine collection test. Those patients with more than 2 grams of proteinuria during the 24-hour period should have bevacizumab treatment withheld, and treatment should be resumed when the protein level is less than 2 grams during a 24 -hour period. If the patient develops nephrotic syndrome, bevacizumab should be discontinued [10].

6.2. Sorafenib. Sorafenib is a multikinase inhibitor that stalls tumor growth and angiogenesis by inhibiting intracellular RAF kinases (CRAF, BRAF, and mutant BRAF) and cell surface kinase receptors VEGFR-2, VEGFR-3, platelet-derived growth factor receptor-beta (PDGFR- $\beta$ ), c-kit receptor, and FMS-like tyrosine kinase-3 (FLT3). Sorafenib is metabolized by the liver via the cytochrome P450 3A4 enzyme (CYP3A4) and UGT1A9 (UDP-glucuronosyltransferase 1-9). The halflife elimination is 25 to 48 hours with a time at which plasma level peaks of approximately 3 hours. Sorafenib is administered orally. Monitoring the patient's BP weekly is recommended during the first 6 weeks of sorafenib therapy. Other cardiovascular adverse effects of sorafenib include cardiac ischemia/infarction $(\leq 3 \%)[11]$.
6.3. Sunitinib. Sunitinib exhibits antitumor and antiangiogenic properties by inhibiting multiple receptor tyrosine kinases, including PDGFR- $\alpha$ and PDGFR- $\beta$; VEGFR1, VEGFR-2, and VEGFR-3; FLT3; colony-stimulating factor type-1; glial cell line-derived neurotrophic factor receptor. Sunitinib is metabolized by the liver, predominantly by CYP3A4. The half-life elimination period is 40 to 60 hours with time to peak in plasma of 6 to 12 hours. Sunitinib is administered orally [12]. Cardiac parameters recommended for monitoring include the left ventricular ejection fraction, cardiac risk factors, and electrocardiographic data at baseline and periodically thereafter, along with BP. Adverse cardiovascular effects include a decrease in left ventricular ejection fraction $(11 \%-21 \%$ of patients), heart failure $(\leq 15 \%)$, peripheral edema $(11 \%)$, venous thrombotic events $(2 \%-3 \%)$, and myocardial ischemia (1\%). Patients with an ejection fraction of less than $50 \%$ and greater than $20 \%$ below baseline but without symptoms of heart failure should have the sunitinib dose reduced or the treatment interrupted. Sunitinib should be discontinued in those patients who develop symptoms of heart failure. Sunitinib should also be discontinued in patients who develop severe hypertension, though treatment may be restarted after the hypertension is controlled. Patients are at increased risk of atypical rapid ventricular tachycardia, or torsades de pointes, and if they have any of the following: taking antiarrhythmic medications, prolonged QT interval syndrome, preexisting bradycardia, and underlying cardiac disease [12].

6.4. Nilotinib. Nilotinib is an inhibitor of the Bcr-Abl kinase that binds and stabilizes the inactive conformation of the kinase domain of the Abl protein, which inhibits proliferation of leukemic cell lines in patients with the Philadelphia chromosome $(\mathrm{Ph})$ associated with chronic myelogenous leukemia (CML). Nilotinib is metabolized by the liver via CYP3A4 to inactive metabolites with a half-life 
elimination period of 15 to 17 hours. Nilotinib is administered orally. Nilotinib can prolong the QTc interval and predispose patients to torsades de pointes and sudden death [13]. Using nilotinib in patients with a QTc interval $>480$ milliseconds is contraindicated; in those patients, prompt attention and correction of electrolyte abnormalities (such as hypokalemia and hypomagnesemia) are warranted [13]. Medications that prolong the QTc interval, such as amiodarone hydrochloride, disopyramide phosphate, dofetilide, ibutilide fumarate, procainamide hydrochloride, quinidine, sotalol hydrochloride, haloperidol, methadone hydrochloride, levofloxacin, and clarithromycin should be avoided or substituted when nilotinib is used [21]. Avoid concomitant administration of CYP3A4 inhibitors such as ketoconazole, voriconazole, itraconazole, clarithromycin, ritonavir, and grapefruit products. Patients may resume nilotinib within 2 weeks at the previous dose if their QTc interval returns to $<450$ milliseconds and is within 20 milliseconds of the baseline. If their QTc interval is between 450 and 480 milliseconds after 2 weeks, the nilotinib dose should be reduced. An electrocardiogram (ECG) should be repeated approximately 7 days after any dose adjustments. Other cardiovascular complications of nilotinib are peripheral edema $(>10 \%)$, angina, chest pain, hypertension, and pericardial effusion in $1 \%-10 \%$ of patients [13].

6.5. Pazopanib. Pazopanib is a multityrosine kinase inhibitor of VEGFR-1, VEGFR-2, VEGFR-3, PDGFR- $\alpha$ and $-\beta$, fibroblast growth factor receptor-1 and -3, cytokine receptor (kit), interleukin-2 receptor inducible T-cell kinase, leukocytespecific protein tyrosine kinase, and transmembrane glycoprotein receptor kinase [14].

Pazopanib disrupts angiogenesis in vivo by inhibiting VEGF-induced VEGFR-2 phosphorylation. Pazopanib is administered orally and is primarily metabolized via the CYP3A4 (major) and CYP1A2 and CYP2C8 (minor) pathways. The half-life elimination period of pazopanib is approximately 30 hours. Prolonged QT interval syndrome and torsades de pointes have been reported with use of pazopanib. A QTc interval $>500$ milliseconds has been reported in $1 \%$ of patients with renal cell carcinoma [14]. Torsades de pointes has been reported in less than $1 \%$ of patients treated with pazopanib [14]. Drugs that are CYP3A4 inhibitors (such as ketoconazole, ritonavir, and clarithromycin) may increase pazopanib concentrations, which may increase the risk of arrhythmias. Patients on such medication combinations or those on medications that prolong the QTc interval should be monitored closely for ECG changes, and the pazopanib dose should be modified as needed. A baseline ECG and serum electrolyte analysis are required for patients on pazopanib. Other cardiovascular complications of pazopanib include chest pain $(5 \%$ of patients), myocardial infarction and ischemia (2\%), transient ischemic attack (1\%), and hypertension (overall: $40 \%$; grade 3: 4\%) [14]. Pazopanib should be avoided in patients with evidence of cardiovascular events. In clinical trials of renal cell carcinoma, the incidence of proteinuria was reported at $8 \%$ (grade $3:<1 \%$, and grade $4:<1 \%$ ). Baseline
TABle 2: Common terminology criteria for adverse events v3.0 (CTCAE): hypertension adult.

\begin{tabular}{ll}
\hline Grade & \\
\hline 1 & $\begin{array}{l}\text { Asymptomatic, transient }(<24 \mathrm{hrs}) \text { increase by } \\
>20 \mathrm{~mm} \mathrm{Hg} \text { (diastolic) or to }>150 / 100 \text { if previously } \\
\text { WNL; intervention not indicated }\end{array}$ \\
\hline 2 & $\begin{array}{l}\text { Recurrent or persistent }(\geq 24 \mathrm{hrs}) \text { or symptomatic } \\
\text { increase by }>20 \mathrm{~mm} \mathrm{Hg} \text { (diastolic) or to }>150 / 100 \text { if } \\
\text { previously WNL; monotherapy may be indicated }\end{array}$ \\
\hline 3 & $\begin{array}{l}\text { Requiring more than one drug or more intensive } \\
\text { therapy than before }\end{array}$ \\
\hline 4 & Life-threatening consequences (e.g., hypertensive crisis) \\
\hline 5 & Death \\
\hline $\begin{array}{l}\text { Abbreviations: WNL, within normal limits. } \\
\text { Publication date: August } 9,2006 . \text { Available at: http://ctep.info.nih.gov/ } \\
\text { protocolDevelopment/electronic_applications/docs/ctcaev3.pdf. }\end{array}$
\end{tabular}

and periodic urinalysis to detect the presence of protein should be performed and if grade 4 proteinuria develops, pazopanib should be discontinued [14].

6.6. Dasatinib. Dasatinib inhibits the following kinases: BCR-Abl, Src family (Src, Lck, Fyn), c-kit, EphA2, and PGDFR- $\beta$. On the basis of modeling studies, dasatinib is predicted to bind the multiple conformations of the Abl kinase [15]. Dasatinib is administered orally. Dasatinib is extensively metabolized via CYP3A4 and has a 3- to 5hour elimination half-life period [15]. Some of dasatinib's major cardiovascular complications include fluid retention (21\%-35\% of patients; grades 3, 4: $4 \%-8 \%)$ and pericardial effusion $(<3 \%$; grades $3,4:<3 \%)$. Heart failure and cardiac dysfunction (cardiomyopathy, diastolic dysfunction, decreased ejection fraction, ventricular dysfunction, chest pain, ventricular arrhythmia, and hypertension) have been reported in less than $4 \%$ of patients treated with dasatinib. Dasatinib may prolong the QTc interval, with a reported incidence of less than $1 \%$. Patients with hypokalemia or hypomagnesemia and those taking an antiarrhythmic medication should be closely monitored while on dasatinib therapy [15].

\section{Diagnosis of Hypertension}

In cancer trials, hypertension is commonly classified as an adverse event according to the Common Terminology Criteria for Adverse Events (CTCAE) of the National Cancer Institute and is based on the presence of symptoms, high $\mathrm{BP}$, and need for antihypertensive therapy (Table 2) [22]. A commonly used treatment guideline is that of The Seventh Report of the Joint National Committee on Prevention, Detection, Evaluation, and Treatment of High Blood Pressure (JNC 7) [23]. In this classification system, BP is categorized as normal, prehypertension, or hypertension (Table 3). Thus, CTCAE aids in classifying hypertension as an adverse event or caused by the VEGF inhibitor whereas JNC 7 is used to define and categorize hypertension throughout 
the administration of the VEGF inhibitor. A patient receiving a VEGF inhibitor may have a normal $\mathrm{BP}$ reading when the drug is initiated on the basis of JNC 7 criteria and then may develop prehypertension or stage 1 or 2 hypertension during treatment. If the patient develops stage 1 or 2 hypertension following a normal baseline $\mathrm{BP}$, his/her hypertension will be classified as grade 1 or higher on the CTCAE scale, depending on the duration of the BP increase, the development of symptoms, the degree of $\mathrm{BP}$ increase from the baseline reading, or the need for multidrug antihypertensive therapy.

According to JNC 7, three objectives should be addressed when evaluating patients with hypertension [23]. These objectives include identifying possible etiologies of hypertension, assessing lifestyle (including identifying cardiovascular risk factors or other comorbid conditions that may affect prognosis or treatment of hypertension), and evaluating the presence or absence of hypertension-induced target organ damage.

\section{Timing and Duration of Hypertension Associated with VEGF inhibitors}

The temporal relationship between the initiation of VEGF inhibitors and the onset of hypertension should be carefully examined. A report by Yang et al. [24] noted that the median interval from the first dose of high-dose bevacizumab to the onset of hypertension was 131 days (range: 7-316 days). Johnson et al. reported that the largest increase in systolic pressure was between 7 to $14 \mathrm{~mm} \mathrm{Hg}$ on day 42 of bevacizumab treatment [25]. Seven of 12 patients with major increases in their BP had pre-existing hypertension [25]. A retrospective chart review of 144 patients treated with bevacizumab found that hypertension occurred in 35\% of patients after a median of 11 weeks [26]. The majority of these patients were subsequently treated with an angiotensinconverting enzyme (ACE) inhibitor for hypertension control.

In a study by Zhu et al., the incidences of hypertension in patients receiving low-dose (3.5 or $7.5 \mathrm{mg} / \mathrm{kg} /$ dose $)$ and high-dose (10 or $15 \mathrm{mg} / \mathrm{kg} /$ dose) bevacizumab ranged from $2.7 \%$ to $32.0 \%$ and from $17.6 \%$ to $36.0 \%$, respectively [27]. Thus, the incidence of hypertension appeared to be dose-dependent, with increased incidence of hypertension in those patients on high-dose bevacizumab. A retrospective chart review to determine the adverse effects of bevacizumab therapy found no significant increased incidence of hypertension in patients $\geq 75$ years old [28]. In a small retrospective study, African Americans were more likely than non-African Americans to develop hypertension with bevacizumab treatment [29]. Another potential risk for bevacizumab-induced hypertension is high-dose treatment in patients with underlying renal cell carcinoma; the patients in this study had an overall hypertension incidence from $26 \%$ to $36 \%[24,30]$. In previous clinical trials, bevacizumabinduced hypertension occurred with a BP increase at any time during treatment.

In sunitinib-treated patients, increases in $\mathrm{BP}$ were shown during the second treatment cycle [31] whereas when sorafenib was used there was frequently an increase in BP
TABLE 3: Joint National Commission 7 (JNC 7) Blood Pressure (BP) Classification.

\begin{tabular}{|c|c|}
\hline $\begin{array}{l}\text { Normal BP } \\
\text { Systolic: }<120 \mathrm{~mm} \mathrm{Hg} \text {; } \\
\text { Diastolic: }<80 \mathrm{~mm} \mathrm{Hg}\end{array}$ & \\
\hline $\begin{array}{l}\text { Prehypertension } \\
\text { Systolic: } 120-139 \mathrm{~mm} \mathrm{Hg} \text {; } \\
\text { Diastolic: } 80-89 \mathrm{~mm} \mathrm{Hg}\end{array}$ & $\begin{array}{l}\text { Treatment required in } \\
\text { high-risk cardiovascular } \\
\text { patients }\end{array}$ \\
\hline $\begin{array}{l}\text { Stage } 1 \text { hypertension } \\
\text { Systolic: } 140-159 \mathrm{~mm} \mathrm{Hg} \text {; } \\
\text { Diastolic: } 90-99 \mathrm{~mm} \mathrm{Hg}\end{array}$ & Treatment required \\
\hline $\begin{array}{l}\text { Stage } 2 \text { hypertension } \\
\text { Systolic: } \geq 160 \mathrm{~mm} \mathrm{Hg} ; \\
\text { Diastolic: } \geq 100 \mathrm{~mm} \mathrm{Hg}\end{array}$ & Treatment required \\
\hline
\end{tabular}

( $\geq 20 \%$ systolic and $\geq 10 \%$ diastolic) after 3 weeks of treatment, with a BP plateau afterwards [32]. In a phase 2 open-label study of patients with Philadelphia chromosomepositive CML in the chronic phase following imatinib resistance and intolerance, Kantarjian et al. reported the incidences of pleural effusion and fluid retention as $1 \%$ and very rare $(<1 \%)$, respectively, in association with nilotinib. In the same study, prolongation of the QTc interval $>500$ milliseconds was observed in $0.7 \%$ of patients [33].

In a phase 2 study of cancer patients with metastatic or recurrent invasive breast cancer treated with pazopanib, 52\% of patients had hypertension (any grade); the incidence of grade 1 or 2 hypertension was $38 \%$ and grade 3 hypertension was $14 \%$. In this study, the authors concluded that development of hypertension might be a predictor of response to the angiogenesis inhibitor, as 9 (75\%) of the 12 patients who had stable disease or a partial response developed hypertension whereas only $3(43 \%)$ of the 7 who had progressive disease developed hypertension $(P=.33)$. The time to progression was longer in patients who developed hypertension than in those who did not (5.3 months versus 1.7 months, resp.). This study did not provide any statistical analysis for the time to progression in the two groups (with and without hypertension) during treatment with pazopanib. Therefore, the role of hypertension in the response to pazopanib therapy needs to be validated [34].

In patients treated with dasatinib, the risk of pleural effusion, pericardial effusion, and severe pulmonary edema may be related to the dose they receive. In a study of 138 patients with CML, Quintás-Cardama et al. reported a 35\% overall incidence of pleural effusion (grades 3, 4 in 17\% of patients). The researchers used multivariate analysis to determine that a history of cardiac disease, hypertension, and use of a twice-daily dosing schedule (versus once daily) were risk factors associated with the development of pleural effusions. The authors suggested that the following could be used to manage cardiovascular complications associated with dasatinib therapy: treatment interruption, changing the dosing interval to daily, management of hypertension, and the judicious use of diuretics, pulse steroids, and in severe cases, thoracentesis [35]. 


\section{Intervention and Treatment of VEGF Inhibitor-Induced Hypertension}

At present, there are no evidence-based guidelines or expert consensus on the treatment of VEGF inhibitor-induced hypertension differing from the JNC 7 guidelines. However, all patients should have optimal BP control prior to initiating treatment with any of the VEGF inhibitors.

Recommendations for bevacizumab caution its use in patients with preexisting hypertension and advise close monitoring of $\mathrm{BP}$ (at least every 2 to 3 weeks during treatment) in all patients. Permanent discontinuation of bevacizumab is recommended in patients who develop hypertensive crisis or encephalopathy. For patients who develop uncontrolled hypertension, therapy should be discontinued, at least temporarily. There is no recommendation to continue to monitor BP after therapy is discontinued, although it may take several weeks for the drug to be eliminated [10].

It is recommended that patients receiving sorafenib have weekly BP readings taken during the first 6 weeks of therapy, with periodic monitoring thereafter. Caution should be used when prescribing sorafenib for patients with poorly controlled hypertension [11].

Patients treated with sunitinib should have their therapy interrupted if their hypertension is uncontrolled or severe until the hypertension can be controlled. The BP of those patients on sunitinib should be monitored closely if they have been diagnosed previously with hypertension or poorly controlled hypertension [12]. Also, patients treated with nilotinib, pazopanib, or dasatinib should be monitored closely for hypertension [13-15]. Any home monitoring devices should be validated with clinic devices so that accurate readings are recorded; this process ensures confidence in basing treatment interventions on readings taken at either location (at home or in the clinic). All patients receiving VEGF inhibitors should be encouraged to monitor their BP at home. The frequency of the monitoring should depend on whether the patient has preexisting hypertension, the level of BP control prior to initiation, the dosage and type of VEGF inhibitor, and other comorbidities, especially those that increase cardiovascular risks.

Although there is no specific antihypertensive agent recommended for VEGF inhibitor-induced hypertension, both calcium channel blockers and ACE inhibitors have been used to successfully treat this hypertension. ACE inhibitors have been proposed as a more effective treatment because angiogenesis-induced hypertension mediated through the nitric oxide pathway may be affected by ACE inhibitors but not necessarily by calcium channel blockers [36]. ACE inhibitors may also prevent proteinuria and plasminogen activator inhibitor-1 expression [37]; however, treatment suggestions for VEGF inhibitor-induced hypertension typically follow the JNC 7 guidelines, as previously outlined [23].

VEGF inhibitor-induced hypertension typically requires treatment with more than one antihypertensive agent. Careful BP monitoring with necessary adjustments in antihypertensive medications are essential. Because sorafenib is metabolized through the cytochrome p450 chain, nondihydropyridine calcium channel blockers (verapamil hydrochlo- ride, diltiazem hydrochloride) should not be prescribed in combination with sorafenib; both drugs inhibit the CYP3A4 isoenzyme and may significantly increase sorafenib levels [11]. If calcium channel blockers are necessary for BP control, the preferred choice is amlodipine besylate or nifedipine. In those patients who have an increased risk of developing heart failure and hypertension, medications utilized for the management of heart failure are preferred and are also effective antihypertensive medications (e.g., ACE inhibitors, beta blockers, angiotensin receptor blockers) [23].

A recent prospective clinical trial with cediranib, a selective VEGF signaling inhibitor used in Europe, focused on minimizing dose interruptions and reductions so that the maximum dose intensity of cediranib could be utilized [38]. In this trial, 126 patients with advanced solid tumors were randomized to 1 of 4 groups. The groups included cediranib 30 or $45 \mathrm{mg} / \mathrm{d}$ with or without antihypertensive prophylaxis. All patients who developed hypertension while on cediranib therapy were treated with a standardized, predefined hypertension management algorithm that utilized a low dose of a calcium channel blocker 3 to 7 days prior to initiating cediranib. They reported that overall cediranib was well tolerated and that all groups achieved a high-dose intensity during the first 12 weeks of treatment; antihypertensive prophylaxis did not result in reduced dose reductions or interruptions. Increases in BP were noted early in treatment for all groups and were managed successfully; severe hypertension occurred in only 1 patient receiving prophylaxis versus 18 in the nonprophylaxis groups. The researchers concluded that all four regimens were well tolerated with high-dose cediranib but that no strategy was clearly superior [38]. In previous cediranib studies, there was no preexisting antihypertensive protocol management $[39,40]$. The researchers felt that early diagnosis and treatment of hypertension are likely to decrease the severity of hypertensive events $[39,40]$. Unfortunately there is no published data documenting the time course of VEGF inhibitor-induced hypertension upon withdrawal of the agent. Anecdotally, most patients seem to have improvement of their blood pressure over several weeks to months upon withdrawal of the agent.

\section{Future Aspects}

Maitland et al. proposed that increases in BP after treatment with agents targeting VEGF signaling may be an effective biomarker of VEGF signaling inhibition and that dose titration causing an increase in BP may produce better antitumor effects and subsequently improve clinical outcomes in cancer patients as intended [39]. Future prospective studies are needed to validate these preliminary observations. However, there has been discussion that discontinuing these agents because of BP increases may hinder overall cancer treatment.

\section{Summary}

As cancer screening methods and detection rates for common cancers such as breast and colorectal cancer continue to improve, many primary care physicians will likely care for cancer patients being treated with VEGF inhibitors. 
These physicians and other healthcare providers will be asked to help manage their patients' hypertension and other comorbidities. It is imperative that these clinicians be familiar with the fact that hypertension can be induced by VEGF inhibitors. The currently recommended management of VEGF-induced hypertension is similar to the management of hypertension outlined in the JNC 7 guidelines. However, baseline BP measurement should be performed prior to administering any VEGF inhibitor, and elevated BP should be treated if it is not in the normotensive ranges recommended by the JNC 7 criteria. All patients should have careful $\mathrm{BP}$ monitoring during treatment, and aggressive BP treatment should be instituted when increases in BP are noted. Monitoring should be continued after treatment completion in those patients who have increased BP during treatment until the BP normalizes [41]. Treatment selections include ACE inhibitors and calcium channel blockers, but that decision should be made on the basis of individual patients' characteristics, the type of VEGF agent used, and other coexisting comorbidities. Those patients with undiagnosed hypertension at baseline should be diagnosed and treated appropriately before VEGF-inhibitor therapy is initiated.

As research continues to seek newer, more effective classes of pharmaceuticals to treat cancer, other harmful side effects like hypertension may be discovered. Careful reporting of adverse events is needed so that treatment-associated events can be recognized and promptly addressed with appropriate interventions so that oncologic interventions can be administered safely.

\section{Key Aspects}

(i) VEGF inhibitors utilized in oncologic care in the United States include bevacizumab (Avastin), sorafenib (Nexavar), sunitinib (Sutent), nilotinib (Tasigna), pazopanib (Votrient), and dasatinib (Sprycel).

(ii) VEGF inhibitors frequently increase BP readings during treatment.

(iii) Patients with hypertension and other cardiac comorbidities should be carefully assessed prior to initiating treatment with VEGF inhibitors.

(iv) Baseline BP readings should be taken prior to VEGF inhibitor treatment, and those patients with prehypertension and undiagnosed hypertension should be started on appropriate anti-hypertensive management.

(v) Patients with uncontrolled hypertension prior to initiating treatment with VEGF inhibitors should undergo appropriate evaluation and treatment so that BP control is achieved.

(vi) At this time, the JNC 7 guidelines for diagnosis and management of hypertension are appropriate for VEGF-induced hypertension. (vii) ACE inhibitors and calcium channel blockers have been utilized to manage VEGF-induced hypertension. However, no significant evidence exists to support these selections, and, therefore, hypertension treatment selections should be made on the basis of JNC 7 recommendations.

(viii) Multiple antihypertensives may be necessary for some patients with VEGF-induced hypertension.

(ix) Posttreatment (no longer being treated with a VEGF agent) monitoring may be necessary for those patients who develop hypertension while on treatment.

(x) Treatment should be discontinued in patients who develop malignant hypertension.

(xi) Treatment should be discontinued in patients who develop severe hypertension until appropriate hypertension management is attained.

(xii) Some VEGF inhibitors can prolong the QTc interval. Periodic ECG monitoring is required in patients with a history of, or who are at risk of, prolonged QT interval syndrome (those patients with electrolyte abnormalities or those patients on multiple medications that can induce or inhibit VEGF inhibitors).

(xiii) Because some VEGF inhibitors can cause fluid retention in patients, assessment of cardiopulmonary function is required.

\section{Acknowledgment}

The authors thank Kristi M. Speights for her editorial assistance.

\section{References}

[1] J. Folkman, "Angiogenesis in cancer, vascular, rheumatoid and other disease," Nature Medicine, vol. 1, no. 1, pp. 27-31, 1995.

[2] I. J. Fidler and L. M. Ellis, "The implications of angiogenesis for the biology and therapy of cancer metastasis," Cell, vol. 79, no. 2, pp. 185-188, 1994.

[3] J. Folkman, "Tumor angiogenesis: therapeutic implications," The New England Journal of Medicine, vol. 285, no. 21, pp. 1182-1186, 1971.

[4] D. M. M and P. Baluk, "Significance of blood vessel leakiness in cancer," Cancer Research, vol. 62, no. 18, pp. 5381-5385, 2002.

[5] D. Hanahan and J. Folkman, "Patterns and emerging mechanisms of the angiogenic switch during tumorigenesis," Cell, vol. 86, no. 3, pp. 353-364, 1996.

[6] G. Bergers and L. E. Benjamin, "Tumorigenesis and the angiogenic switch," Nature Reviews Cancer, vol. 3, no. 6, pp. 401410, 2003.

[7] R. Longo, R. Sarmiento, M. Fanelli, B. Capaccetti, D. Gattuso, and G. Gasparini, "Anti-angiogenic therapy: rationale, challenges and clinical studies," Angiogenesis, vol. 5, no. 4, pp. 237256, 2002.

[8] J. Rak, J. Filmus, and R. S. Kerbel, "Reciprocal paracrine interactions between tumour cells and endothelial cells: the 'angiogenesis progression' hypothesis," European Journal of Cancer Part A, vol. 32, no. 14, pp. 2438-2450, 1996. 
[9] Y. Kakeji and B. A. Teicher, "Preclinical studies of the combination of angiogenic inhibitors with cytotoxic agents," Investigational New Drugs, vol. 15, no. 1, pp. 39-48, 1997.

[10] Bevacizumab (Avastin), Package Insert. Genentech, Inc., http://www.avastin.com/.

[11] Sofafenib (Nexavar), Package Insert. Bayer Healthcare Pharmaceuticals Inc., http://www.nexavar.com/.

[12] Sunitinib (Sutent), Package Insert. Pfizer Inc., http://www .sutent.com/.

[13] Nitolinib (Tasigna), Package Insert. Novartis Pharmaceuticals Corporation, http://www.us.tasigna.com/.

[14] Pazopanib (Votrient), Package Insert. GlaxoSmithKlien, http://www.votrient.com/.

[15] Dasitinib (Sprycel), Package Insert. Bristol-Myers Squibb Company, http://www.sprycel.com/.

[16] I. I. H. Chen, R. L. Prewitt, and R. F. Dowell, "Microvascular rarefaction in spontaneously hypertensive rat cremaster muscle," American Journal of Physiology, vol. 10, no. 3, pp. 306-310, 1981.

[17] P. M. Hutchins, C. D. Lynch, P. T. Cooney, and K. A. Curseen, "The microcirculation in experimental hypertension and aging," Cardiovascular Research, vol. 32, no. 4, pp. 772780, 1996.

[18] N. Steeghs, H. Gelderblom, J. O. Roodt et al., "Hypertension and rarefaction during treatment with telatinib, a Small molecule angiogenesis inhibitor," Clinical Cancer Research, vol. 14, no. 11, pp. 3470-3476, 2008.

[19] M. L. Veronese, A. Mosenkis, K. T. Flaherty et al., "Mechanisms of hypertension associated with BAY 43-9006," Journal of Clinical Oncology, vol. 24, no. 9, pp. 1363-1369, 2006.

[20] C. Wheeler-Jones, R. Abu-Ghazaleh, R. Cospedal, R. A. Houliston, J. Martin, and I. Zachary, "Vascular endothelial growth factor stimulates prostacyclin production and activation of cytosolic phospholipase $\mathrm{A}_{2}$ in endothelial cells via p42/p44 mitogen-activated protein kinase," FEBS Letters, vol. 420, no. 1, pp. 28-32, 1997.

[21] D. M. Roden, "Drug-induced prolongation of the QT interval," The New England Journal of Medicine, vol. 350, no. 10, pp. 1013-1022, 2004.

[22] Cancer Therapy Evaluation Program, "Common Terminology Criteria for Adverse Events,” V3.0, DCTD, NCI, NIH, DHHs. March 2003, http://ctep.cancer.gov/.

[23] A. V. Chobanian, G. L. Bakris, H. R. Black et al., "The seventh report of the Joint National Committee on Prevention, Detection, Evaluation, and Treatment of High Blood Pressure: the JNC 7 report," Journal of the American Medical Association, vol. 289, no. 19, pp. 2560-2572, 2003.

[24] J. C. Yang, L. Haworth, R. M. Sherry et al., "A randomized trial of bevacizumab, an anti-vascular endothelial growth factor antibody, for metastatic renal cancer," The New England Journal of Medicine, vol. 349, no. 5, pp. 427-434, 2003.

[25] D. H. Johnson, L. Fehrenbacher, W. F. Novotny et al., "Randomized phase II trial comparing bevacizumab plus carboplatin and paclitaxel with carboplatin and paclitaxel alone in previously untreated locally advanced or metastatic non-small-cell lung cancer," Journal of Clinical Oncology, vol. 22, no. 11, pp. 2184-2191, 2004.

[26] A. U. Pande, J. C. Lombardo, M. Fakih et al., "Bevacizumab induced hypertension: a manageable toxicity (abstract)," Journal of Clinical Oncology, vol. 24, p. 13539, 2006.

[27] X. Zhu, S. Wu, W. L. Dahut, and C. R. Parikh, "Risks of proteinuria and hypertension with bevacizumab, an antibody against vascular endothelial growth factor: systematic review and meta-analysis," American Journal of Kidney Diseases, vol. 49, no. 2, pp. 186-193, 2007.
[28] A. K. Raman, J. C. Lombardo, R. Chandrasekhar et al., "Bevacizumab related adverse events among various age groups of elderly patients with advanced colorectal cancer (abstract)," Journal of Clinical Oncology, vol. 25, pp. 145-146, 2007.

[29] Y. M. Choi, S. Shord, S. Cuellar et al., "Examining ethnic differences for bevacizumab-induced hypertension and proteinuria (abstract)," Journal of Clinical Oncology, vol. 25, p. 21168, 2007.

[30] B. Escudier, A. Pluzanska, P. Koralewski et al., "Bevacizumab plus interferon alfa-2a for treatment of metastatic renal cell carcinoma: a randomised, double-blind phase III trial," The Lancet, vol. 370, no. 9605, pp. 2103-2111, 2007.

[31] M. Azizi, A. Chedid, and S. Oudard, "Home blood-pressure monitoring in patients receiving sunitinib," The New England Journal of Medicine, vol. 358, no. 1, pp. 95-97, 2008.

[32] M. L. Veronese, K. T. Flahert, R. Townsend et al., "Pharmacodynamic study of the rat kinase inhibitor BAY 43-9006: mechanism of hypertension," Journal of Clinical Oncology, vol. 22, supplement: 135 poster no. 2035, 2004.

[33] H. M. Kantarjian, F. Giles, N. Gattermann et al., "Nilotinib (formerly AMN107), a highly selective BCR-ABL tyrosine kinase inhibitor, is effective in patients with Philadelphia chromosome-positive chronic myelogenous leukemia in chronic phase following imatinib resistance and intolerance," Blood, vol. 110, no. 10, pp. 3540-3546, 2007.

[34] S. K. Taylor, S. Chia, S. Dent et al., "A phase II study of pazopanib in patients with recurrent or metastatic invasive breast carcinoma: a trial of the Princess margaret hospital phase II Consortium," The Oncologist, vol. 15, no. 8, pp. 810$818,2010$.

[35] A. Quintás-Cardama, H. Kantarjian, S. O’Brien et al., "Pleural effusion in patients with chronic myelogenous leukemia treated with dasatinib after imatinib failure," Journal of Clinical Oncology, vol. 25, no. 25, pp. 3908-3914, 2007.

[36] H. I. Hurwitz, L. Fehrenbacher, J. D. Hainsworth et al., "Bevacizumab in combination with fluorouracil and leucovorin: an active regimen for first-line metastatic colorectal cancer," Journal of Clinical Oncology, vol. 23, no. 15, pp. 3502-3508, 2005.

[37] M. Dincer and K. Altundag, "Angiotensin-converting enzyme inhibitors for bevacizumab-induced hypertension," Annals of Pharmacotherapy, vol. 40, no. 12, pp. 2278-2279, 2006.

[38] M. H. G. Langenberg, C. M. L. Van Herpen, J. De Bono et al., "Effective strategies for management of hypertension after vascular endothelial growth factor signaling inhibition therapy: results from a phase II randomized, factorial, doubleblind study of cediranib in patients with advanced solid tumors," Journal of Clinical Oncology, vol. 27, no. 36, pp. 61526159, 2009.

[39] M. L. Maitland, K. Moshier, J. Imperial et al., "Blood pressure as a biomarker for sorafenib, an inhibitor of the VEGF signaling pathway," Journal of Clinical Oncology, vol. 24: 87s, supplement; abstract 2035, 2006.

[40] M. L. Maitland and M. J. Ratain, "Terminal ballistics of kinase inhibitors: there are no magic bullets," Annals of Internal Medicine, vol. 145, no. 9, pp. 702-703, 2006.

[41] M. L. Maitland, G. L. Bakris, H. R. Black et al., "Initial assessment, surveillance, and management of blood pressure in patients receiving vascular endothelial growth factor signaling pathway inhibitors," Journal of the National Cancer Institute, vol. 102, no. 9, pp. 596-604, 2010. 


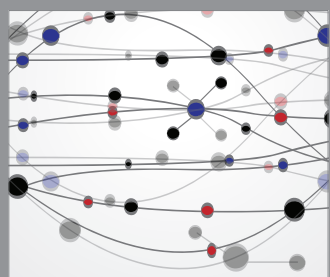

The Scientific World Journal
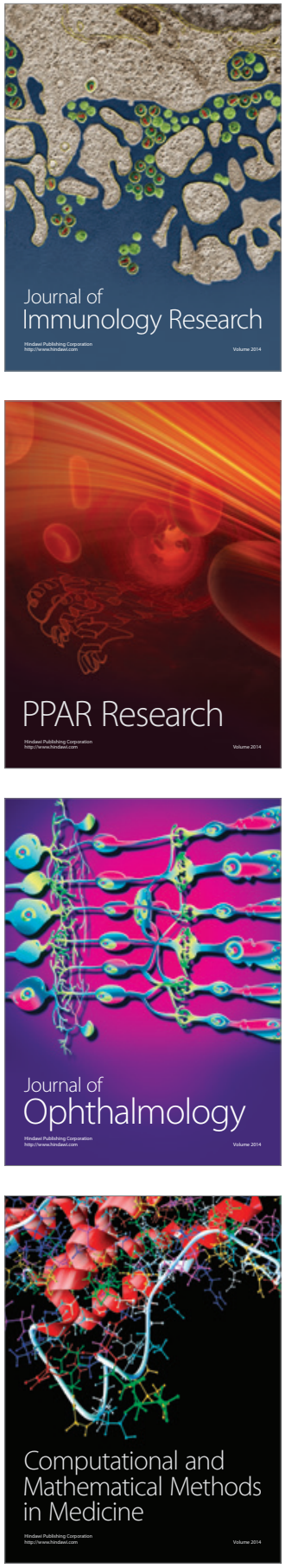

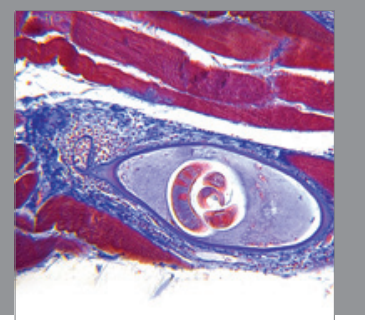

Gastroenterology

Research and Practice
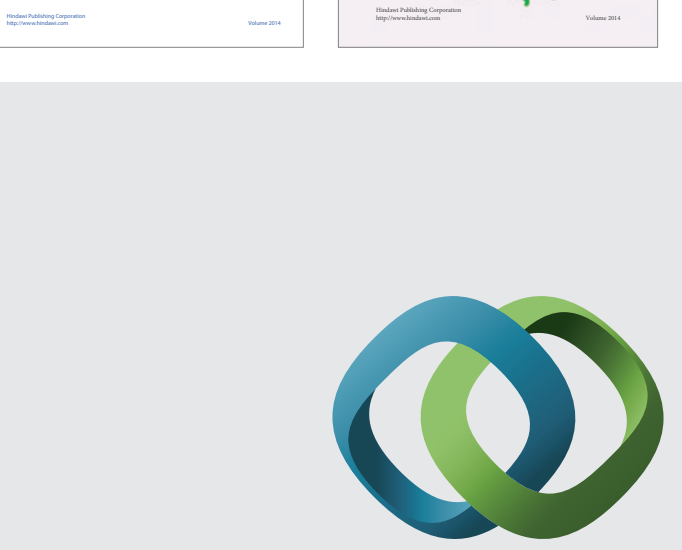

\section{Hindawi}

Submit your manuscripts at

http://www.hindawi.com
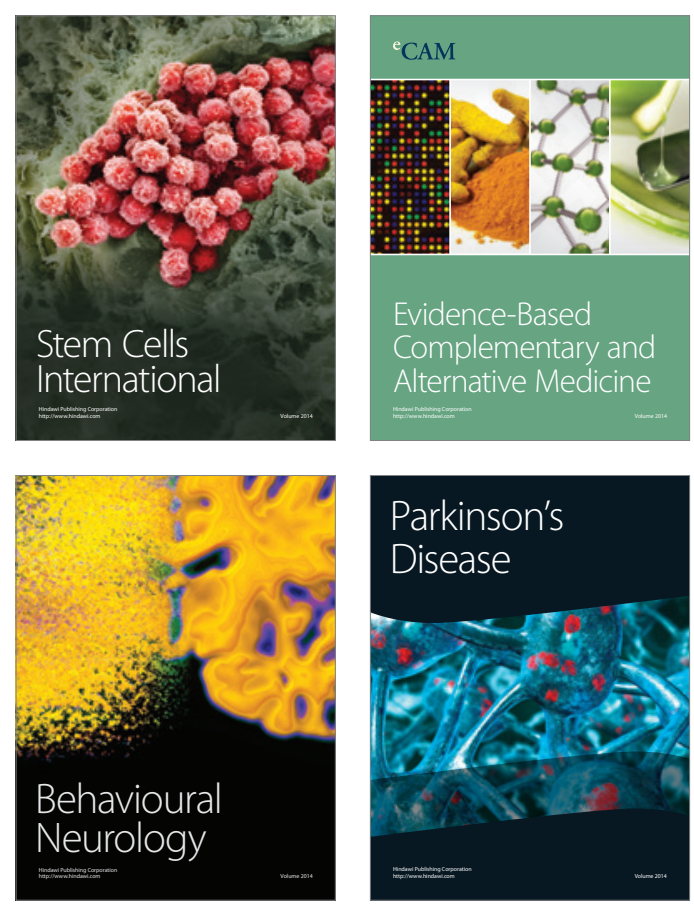

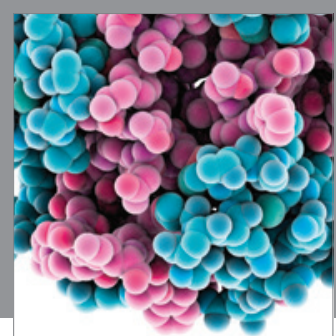

Journal of
Diabetes Research

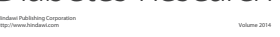

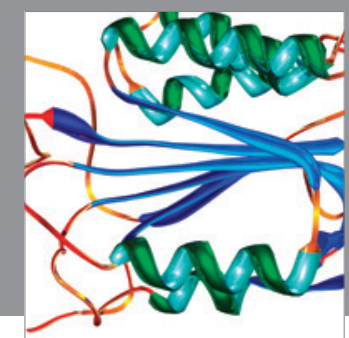

Disease Markers
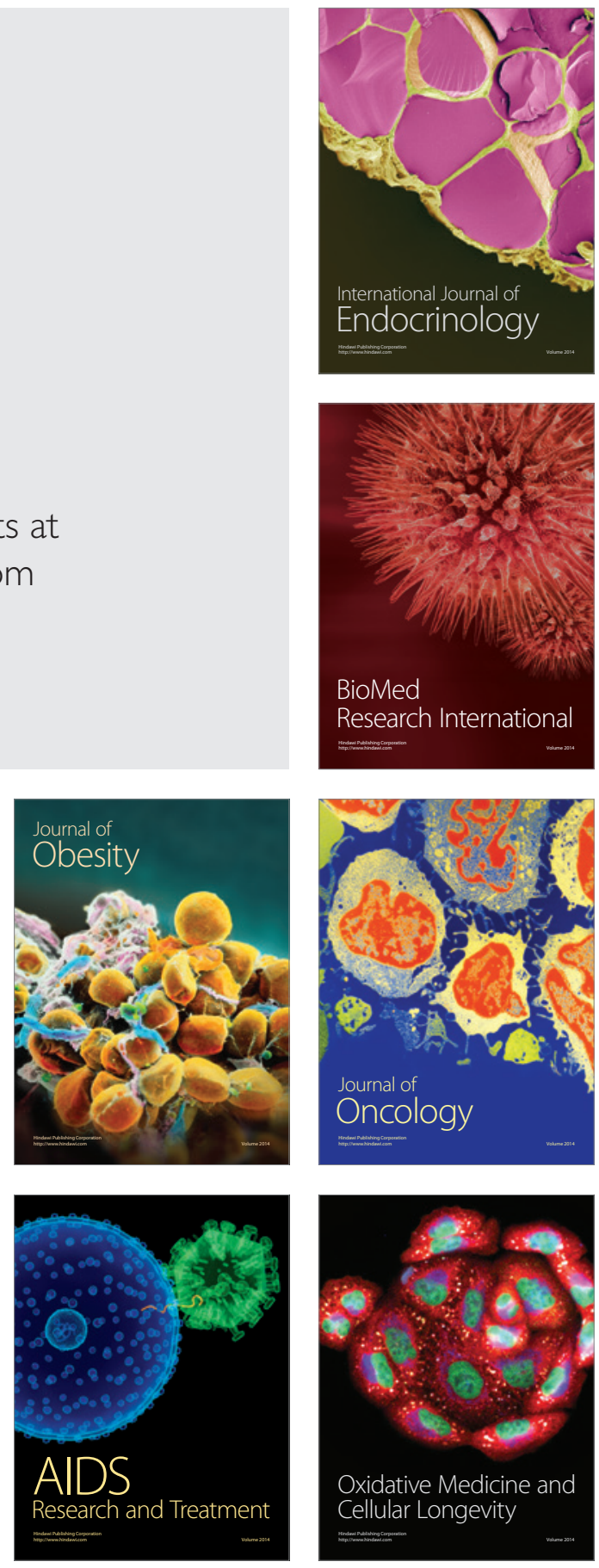